\title{
Diatoms (Bacillariophyta) from the Genus Eunotia and Pinnularia Developing on Soils in the Open Landscape of the Low Beskids
}

\author{
Anita Poradowska ${ }^{1}$ \\ 1 Department of Landscape Architecture, Institute of Agricultural Sciences, Land Management and \\ Environmental Protection, University of Rzeszów, Ćwiklińskiej 1A, 35-601 Rzeszów, Poland \\ e-mail: anita.pajaczek@gmail.com
}

\begin{abstract}
In the areas of medium mountains, including the landscape of the Low Beskids, turf communities guarantee the biodiversity increasing the value of open landscape and protecting soil from erosion. The aim of this study was to show the diversity of the diatom communities from the Eunotia and Pinnularia genera developing on soils under the conditions of varied landscape use (meadows, pastures, fallow lands). The research was carried out within the catchment area of the Ryjak stream in the Magura National Park, together with the buffer zone at three sites. The water content varied in the studied soils with fluctuating moisture content depending on the season and the research year. On the other hand, the $\mathrm{pH}$ values indicated high acidification of the studied soils. The diatomaceous analysis showed numerous occurrences of the Eunotia (12 taxa) and Pinnularia (18 taxa) genera. The acid pH of the soil undoubtedly favored the development of diatoms of these genera. Most species of the Eunotia and Pinnularia genera usually developed individually, except for Pinnularia borealis Ehrenberg and P. obscura Krasske. They are typically soil species; therefore, they often dominated in the material and occurred in most of the research seasons. Apart from cosmopolitan diatoms, rare or endangered taxa from the Red List of the algae in Poland (Siemińska et al. 2006) were also found on soils. Four species belonged to the endangered category (E): Eunotia botuliformis F. Wild, Nörpel \& Lange-Bert., Pinnularia nodosa (Ehrenberg) Smith, P. schoenfelderi and P. viridiformis Krammer. Canonical analysis (CCA) demonstrated a rather low statistical significance of the impact of environmental parameters ( $\mathrm{pH}$ and humidity) on the differentiation of diatom communities. It only confirmed the fact that diatoms develop better in the environment with permanent or at least periodic moisture, compared to the dry habitats.
\end{abstract}

Keywords: soil diatoms, diversity

\section{INTRODUCTION}

The open landscape, which is generally the case of the Low Beskids, was created by the natural forces and as such is also subjected to slow, natural transformations. The human activity resulting from e.g. construction of rural settlements, farming, etc. also has slight impact on its transformations (Patoczka 2016). The most of agricultural lands in the Low Beskids and in the entire Polish Carpathians are located along the river valleys. Turf assemblages in river valleys guarantee biodiversity, improve the landscape values and have a protective function against surface runoff (Żyszkowska 2003, Jankowska-Huflejt 2007). The characteristic soil types forming the landscape of the middle mountains (including the Low Beskids) are the initial soils, the occurrence of which is, however, mostly limited to dorsal rocky outcrops. The soil cover structure is dominated by brown soils, among them eutrophic brown soils (Skiba 2006).

Soil algae are very important group of soil microorganisms that can be used as an excellent tool for testing the quality of the environment. Due to the large amplitude of adaptation to changing environmental conditions, they are good indicators of environmental quality and their activity is very important for soil functioning (Metting 1981, Gajda \& Przewłoka 2012).

The algae in terrestrial habitats have been under research worldwide for many years. Attempts 
are being made to use algae as indicators of the changes in the soil environment resulting from the use of fertilizers, pesticides, tillage systems and varieties of arable crops (Gollerbach \& Sztina 1969, Sieminiak 1998, 2003, Zancan et al. 2006, Adesalu et al. 2015). Many scientific works concern the structure and species composition of cyanobacteria and green algae (Durrel 1959, 1962, Cameron 1960, 1962, Shields \& Drouet 1962, Hunt \& Durrell 1966, Komárek \& Fott 1983, Ettl \& Gärtner 1995, Johansen \& Shubert 2001, Zancan et al. 2006). Likewise, there are few studies on the taxonomy and ecology of soil diatom assemblages, although interest in these microorganisms has been increasing in recent years (Hahn \& Neuhaus 1997, Bérard et al. 2004, Zancan et al. 2006, Al-Fredan \& Fathi 2007, Kalinowska \& Pawlik-Skowrońska 2008, Škaloud 2009, Heger et al. 2012, Antonelli et al. 2017, Pfister et al. 2017, Barragán et al. 2018, Foets et al. 2020).

The research on the diversity of the diatom communities on various types of agricultural soils has also been conducted for several years in south-eastern Poland (Stanek-Tarkowska \& Noga 2012a,b, Stanek-Tarkowska et al. 2013, 2015, 2016, 2017, Noga et al. 2014, 2017). The authors show considerable variability in the structure of the diatom communities depending on the type of soil and its usage.

The aim of this work was to show the diversity of the diatom communities from the Eunotia and Pinnularia genera growing on soils under diverse landscape use (meadows, pastures, fallow lands) in the Low Beskids.

\section{STUDY AREA}

The research was carried out in the Magura National Park with a buffer zone, located in the mountainous landscape of the Low Beskids with rich flora and fauna, usually associated with meadow, pasture and forest habitats. The area is covered by special operating principles defined by a special nature protection zone. The cultural landscape is formed by the remains of former Lemko villages. Numerous churches and remains of residential buildings are a reminiscence of the past culture.

The catchment area of the Ryjak stream is $43.6 \mathrm{~km}^{2}$ and the natural and forest landscape predominates in this area. In the stream valley, there are permanent grasslands, i.e. meadows and pastures, on ranker soils with browning characteristics SQbr (site R1) and on eutrophic brown soil BEgg (sites R2, R3) (Skiba 2006, Szewczyk 2017).

A total of 3 study sites were designated on soils with different uses, within the catchment area of the Ryjak stream: 1) the first site R1 on a pasture (N21,465655; E49,425374), 2) the second one R2 on a meadow (N21,433175; E49,458211) and the third site R3 on fallow land (N21,428535; E49,483552).

The R1 site was exposed and sunny, the soil was covered with low turf (grazed by cattle), stamped with animal hooves. The soil was often dry during the vegetation season. The R2 site was permanently shaded, the soil was overgrown with meadow vegetation (typical for communities in the order Arrhenatheretalia elatioris), mown once a year or less frequently. After heavy rainfall, the groundwater pools overgrowthd with mosses in some places. The R3 site can be described as intermediate between the pasture (R1) and the meadow (R2), with unevenly turf soil (ruderal plants grew in some places, and soil was exposed in other).

\section{METHODS}

The material for diatom research and soil physicochemical analysis was collected from three sites (R1-R3) twice a year (from spring 2013 to spring 2019). The sites were designated on the soils in the catchment area of the upper, middle and lower section of the Ryjak stream. The date and method of soil sampling for the physicochemical analyses was established on the basis of the recommendations of the National Chemical and Agricultural Station in Warsaw (https://schr.gov.pl/).

The soil material was taken from pasture, fallow land and meadow, from a 5-15 cm layer, using a metal spatula, at three randomly selected sites, and an in situ combined sample was created in 1-liter plastic bags. At the same time, the soil samples were taken into $100 \mathrm{~cm}^{3}$ metal cylinders from the surface soil layer $(5-15 \mathrm{~cm})$ to examine the level of soil moisture.

The diatomaceous material was collected randomly at three selected points in each site (exposed, moss-covered, greened), by cutting off a $10 \mathrm{~cm}$ diameter disc to the Petri dish with a selected fragment of the surface soil layer $(0-2 \mathrm{~cm})$. 
Then, a combined sample was prepared in the laboratory, ready for preparation, by taking the samples with a scalpel (about $1 \mathrm{~cm}^{3}$ ). The materials were collected according to the methodology provided by Stanek-Tarkowska \& Noga (2012b) and Stanek-Tarkowska et al. (2013).

In the laboratory, the $\mathrm{pH}$ was measured using the potentiometric method in a soil solution with distilled water $\left(\mathrm{pH}\right.$ in $\left.\mathrm{H}_{2} \mathrm{O}\right)$ (Karczewska \& Kabała 2008) and the percentage moisture content after drying at $105^{\circ} \mathrm{C}$ for 72 hours.

The diatomaceous material was macerated for several days in a mixture of $\mathrm{K}_{2} \mathrm{Cr}_{2} \mathrm{O}_{7}$ potassium dichromate and concentrated sulfuric acid VI $\mathrm{H}_{2} \mathrm{SO}_{4}$ (so-called 'chromianka') in a 3:1 ratio. The material was then centrifuged in a Thermo Scientific Megafuge 8 laboratory centrifuge at 2,500 r $\times \min -1$ for 5 minutes. Microscopic slides were prepared from the obtained solution, which were closed in Pleurax synthetic resin (refractive index 1.75). The method of diatom purification and the preparation of permanent slides were adopted according to Kawecka (2012).

The microscopic slides were determined under a Carl Zeiss Axio Imager A2 light microscope using Nomarski interference differential contrast (DIC) and a 1.4 planapochromatic lens at 1000 $\times$ magnification. The diatom micrographs were taken with using a light microscope (Carl Zeiss AxioCam ICc 5 camera) and a HITACHI SU8010 scanning electron microscope. The diatoms were identified using the following keys: Krammer \& Lange-Bertalot (1986, 1991), Krammer (2000),
Hofmann et al. (2011) and Lange-Bertalot et al. (2011, 2017).

The number of individual diatom taxa was obtained by counting the valves in each preparation, in individual fields of view of the microscope, until a total of about 300 valves was obtained.

On the basis of the Red List of the algae in Poland (Siemińska et al. 2006) species with different degrees of threat were distinguished. The statistical analysis of the impact of environmental variables on the differentiation of communities was performed in the Canoco 5.0.3 program (Ter Braak, Šmilauer 2012).

\section{RESULTS}

The water content in the studied soils of different usage types was variable, and fluctuated from $5.6 \%$ to $61.9 \%$ depending on the season and the research year. In the first site, the moisture content ranged from $10.4 \%$ in spring 2019 to $54.8 \%$ in autumn 2017 , in the second site between $15.5 \%$ in spring 2019 and $61.9 \%$ in autumn 2016, while in the third site - between 5.6\% in spring 2015 and 52.1\% in autumn 2017 (Table 1).

The $\mathrm{pH}$ values indicated high acidification of the studied soils, because most soils were characterized by very acid and acid $\mathrm{pH}(\mathrm{pH}: 4.4-5.6)$. Only at the third site, in the years 2013-2014, the soil had a neutral $\mathrm{pH}$ (pH: 6.2-6.8), Table 1.

During the research carried out in 20132019, on soils with different ways of landscape

Table 1. The values of $\mathrm{pH}$ and soil humidity measured on designated sites (R1-R3) in the Ryjak stream valley in 13 research seasons (2013-2019)

\begin{tabular}{|c|c|c|c|c|c|c|c|}
\hline \multirow{2}{*}{\multicolumn{2}{|c|}{ Year }} & \multicolumn{2}{|c|}{ R1 pasture } & \multicolumn{2}{|c|}{ R2 fresh meadow } & \multicolumn{2}{|c|}{ R3 fallow land } \\
\hline & & \multirow{2}{*}{$\frac{\mathrm{pH}\left[\mathrm{H}_{2} \mathrm{O}\right]}{5.6}$} & \multirow{2}{*}{$\frac{\text { moisture }[\%, v / v]}{34.5}$} & \multirow{2}{*}{$\frac{\mathrm{pH}\left[\mathrm{H}_{2} \mathrm{O}\right]}{5.6}$} & \multirow{2}{*}{$\frac{\text { moisture }[\%, v / v]}{29.1}$} & \multirow{2}{*}{$\frac{\mathrm{pH}\left[\mathrm{H}_{2} \mathrm{O}\right]}{6.5}$} & \multirow{2}{*}{$\frac{\text { moisture }[\%, v / v]}{29.1}$} \\
\hline \multirow{7}{*}{ Spring } & 2013 & & & & & & \\
\hline & 2014 & 5.2 & 27.7 & 5.4 & 53.8 & 6.2 & 29.7 \\
\hline & 2015 & 5.1 & 12.0 & 4.7 & 26.9 & 5.1 & 5.6 \\
\hline & 2016 & 4.4 & 41.0 & 4.4 & 18.4 & 5.3 & 49.2 \\
\hline & 2017 & 4.9 & 32.1 & 4.5 & 46.8 & 5.1 & 30.0 \\
\hline & 2018 & 4.9 & 46.1 & 4.5 & 47.6 & 4.9 & 43.5 \\
\hline & 2019 & 5.4 & 10.4 & 4.9 & 15.5 & 5.3 & 15.0 \\
\hline \multirow{6}{*}{ Autumn } & 2013 & 5.6 & 15.8 & 5.6 & 15.5 & 6.1 & 17.5 \\
\hline & 2014 & 5.8 & 46.8 & 6.3 & 68.5 & 6.8 & 41.7 \\
\hline & 2015 & 4.6 & 44.5 & 4.7 & 48.3 & 5.9 & 52.5 \\
\hline & 2016 & 5.1 & 49.0 & 5.2 & 61.9 & 5.8 & 52.7 \\
\hline & 2017 & 5.3 & 54.8 & 5.3 & 61.0 & 5.2 & 52.1 \\
\hline & 2018 & 5.1 & 50.9 & 4.6 & 53.4 & 5.1 & 52.2 \\
\hline
\end{tabular}


use in the Ryjak stream catchment area, numerous species of the Eunotia (12 taxa) and Pinnularia (18 taxa) genera were found. The third R3 (8 taxa of Eunotia and 14 taxa of Pinnularia) and the second R2 (7 taxa of Eunotia and 13 taxa of Pinnularia) sites characterized the largest species diversity, while the smallest - the first site R1 (3 taxa of Eunotia and 7 taxa of Pinnularia). The highest species diversity of diatoms in both determined genera was found in 2018, especially at sites R2 and R3 (Table 2). Selected diatom microphotographs, both LM and SEM, are presented in Figures 1-3.

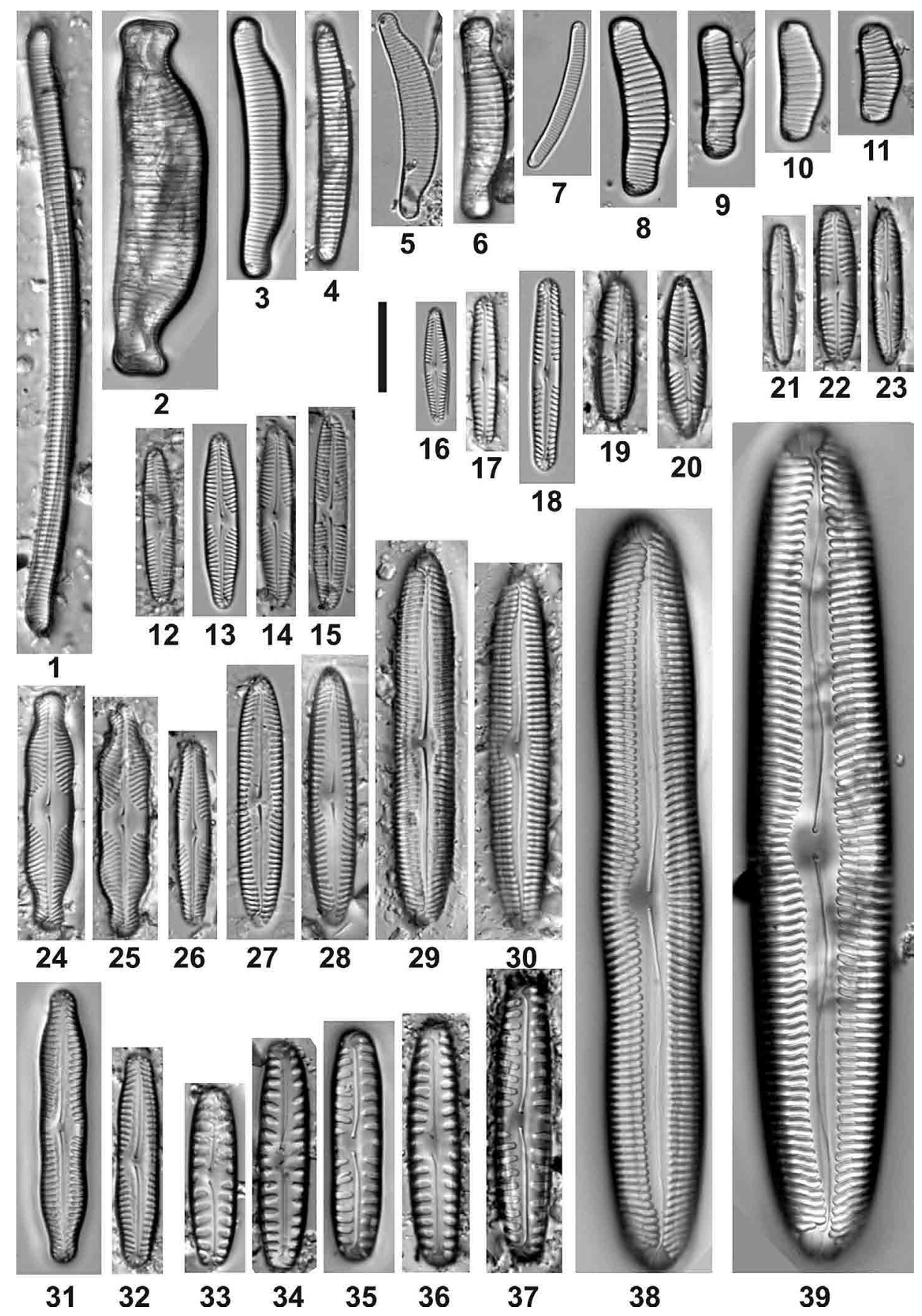

Figure 1. Selected diatom species designated in analyzed materials (LM - microphotographs): 1 - Eunotia cf. glacialifalsa, 2 - E. superbidens, 3 - Eunotia cf. dorofeyukae, 4 -E. valida, 5 - E. ursamaioris, 6 - E. arcubus, 7 - E. botuliformis, 8-11 - E. curtagrunowii, 12, 14, 15 - Pinnularia schoenfelderi, $16 \neg$ P. silvatica, 17, 18 P. sinistra, 19 - P. intermedia, 20 - P. cuneola, 21-23 - P. obscura, 24, 25 - P. grunowii, 13, 26 - P. marchica, 27, 28 - P. isselana, 29, 30 - P. frequentis, 31, 32 - P. nodosa, 33-37 - P. borealis, 38 - P. viridiformis, $39-$ P. viridis. Scale bar $=10 \mu \mathrm{m}$ 
Table 2. The list of taxa and abundance of diatoms from the Pinnularia and Eunotia genus found at individual sites (R1-R3) in 2013-2019 along with cell dimensions (length-width), number of striae (s) and areole (a) in $10 \mu \mathrm{m}$ and categories of threats given in square brackets [according to Siemińska et al. 2006]

\begin{tabular}{|c|c|c|c|c|c|c|c|c|c|c|c|c|c|c|c|c|c|c|c|c|c|}
\hline Year & & 2013 & & & 2014 & & & 2015 & & & 2016 & & & 2017 & & & 2018 & & & 2019 & \\
\hline $\begin{array}{l}\text { Name of taxa } \\
\text { Study sites }\end{array}$ & R1 & $\mathrm{R} 2$ & R3 & R1 & R2 & R3 & R1 & $\mathrm{R} 2$ & R3 & R1 & R2 & R3 & R1 & R2 & R3 & R1 & R2 & R3 & R1 & $\mathrm{R} 2$ & R3 \\
\hline $\begin{array}{l}\text { Eunotia arcubus Nörpel \& } \\
\text { Lange-Bertalot } \\
(31.3 \times 6.4 ; s: 8 \mathrm{n}=1)\end{array}$ & $\begin{array}{l}0 \\
0\end{array}$ & $\begin{array}{l}0 \\
0\end{array}$ & $\begin{array}{l}0 \\
0\end{array}$ & $\begin{array}{l}0 \\
0\end{array}$ & $\begin{array}{l}0 \\
2\end{array}$ & $\begin{array}{l}1 \\
0\end{array}$ & $\begin{array}{l}0 \\
0\end{array}$ & $\begin{array}{l}0 \\
0\end{array}$ & $\begin{array}{l}0 \\
0\end{array}$ & $\begin{array}{l}0 \\
0\end{array}$ & $\begin{array}{l}1 \\
0\end{array}$ & $\begin{array}{l}0 \\
0\end{array}$ & $\begin{array}{l}0 \\
0\end{array}$ & $\begin{array}{l}0 \\
0\end{array}$ & $\begin{array}{l}0 \\
1\end{array}$ & $\begin{array}{l}0 \\
0\end{array}$ & $\begin{array}{l}0 \\
0\end{array}$ & $\begin{array}{l}0 \\
0\end{array}$ & 0 & 0 & 0 \\
\hline $\begin{array}{l}\text { Eunotia botuliformis F. Wild, } \\
\text { Nörpel \& Lange-Bertalot [E] } \\
(14-21.4 \times 2.1-4.1 ; \text { s: } 16-21 ; a \text { : } \\
40-45 ; n=10)\end{array}$ & $\begin{array}{l}0 \\
0\end{array}$ & $\begin{array}{l}0 \\
0\end{array}$ & $\begin{array}{l}0 \\
0\end{array}$ & $\begin{array}{l}0 \\
0\end{array}$ & $\begin{array}{l}3 \\
1\end{array}$ & $\begin{array}{l}0 \\
0\end{array}$ & $\begin{array}{l}0 \\
2\end{array}$ & $\begin{array}{l}1 \\
4\end{array}$ & $\begin{array}{l}0 \\
1\end{array}$ & $\begin{array}{l}0 \\
0\end{array}$ & $\begin{array}{l}5 \\
0\end{array}$ & $\begin{array}{l}0 \\
0\end{array}$ & $\begin{array}{l}0 \\
0\end{array}$ & $\begin{array}{l}0 \\
0\end{array}$ & $\begin{array}{l}0 \\
0\end{array}$ & $\begin{array}{l}0 \\
0\end{array}$ & $\begin{array}{l}0 \\
7\end{array}$ & $\begin{array}{l}0 \\
1\end{array}$ & 0 & 3 & 0 \\
\hline $\begin{array}{l}\text { Eunotia curtagrunowii Nörpel- } \\
\text { Schempp \& Lange-Bertalot } \\
(10.3-29.5 \times 5.2-6.9 ; \text { s: 8-15; a: } \\
34-36 ; \mathrm{n}=11)\end{array}$ & $\begin{array}{l}0 \\
0\end{array}$ & $\begin{array}{l}0 \\
0\end{array}$ & $\begin{array}{l}0 \\
0\end{array}$ & $\begin{array}{l}0 \\
0\end{array}$ & $\begin{array}{c}2 \\
12\end{array}$ & $\begin{array}{l}0 \\
0\end{array}$ & $\begin{array}{l}0 \\
0\end{array}$ & $\begin{array}{l}3 \\
0\end{array}$ & $\begin{array}{l}0 \\
0\end{array}$ & $\begin{array}{l}0 \\
0\end{array}$ & $\begin{array}{c}10 \\
0\end{array}$ & $\begin{array}{l}0 \\
0\end{array}$ & $\begin{array}{l}0 \\
1\end{array}$ & $\begin{array}{c}14 \\
5\end{array}$ & $\begin{array}{l}0 \\
0\end{array}$ & $\begin{array}{l}0 \\
0\end{array}$ & $\begin{array}{c}3 \\
14\end{array}$ & $\begin{array}{l}0 \\
8\end{array}$ & 0 & 1 & 0 \\
\hline Eunotia meisteri Hustedt [l] & $\begin{array}{l}0 \\
0\end{array}$ & $\begin{array}{l}0 \\
0\end{array}$ & $\begin{array}{l}0 \\
0\end{array}$ & $\begin{array}{l}0 \\
0\end{array}$ & $\begin{array}{l}0 \\
0\end{array}$ & $\begin{array}{l}1 \\
0\end{array}$ & $\begin{array}{l}0 \\
0\end{array}$ & $\begin{array}{l}0 \\
0\end{array}$ & $\begin{array}{l}0 \\
0\end{array}$ & $\begin{array}{l}0 \\
0\end{array}$ & $\begin{array}{l}0 \\
0\end{array}$ & $\begin{array}{l}0 \\
0\end{array}$ & $\begin{array}{l}0 \\
0\end{array}$ & $\begin{array}{l}0 \\
0\end{array}$ & $\begin{array}{l}0 \\
0\end{array}$ & $\begin{array}{l}0 \\
0\end{array}$ & $\begin{array}{l}0 \\
0\end{array}$ & $\begin{array}{l}0 \\
0\end{array}$ & 0 & 0 & 0 \\
\hline $\begin{array}{l}\text { Eunotia nymanniana Grunow } \\
(23.7 \times 2.6 ; \mathrm{s} .20 ; \mathrm{n}=1)\end{array}$ & $\begin{array}{l}0 \\
0\end{array}$ & $\begin{array}{l}0 \\
0\end{array}$ & $\begin{array}{l}0 \\
0\end{array}$ & $\begin{array}{l}0 \\
0\end{array}$ & $\begin{array}{l}0 \\
0\end{array}$ & $\begin{array}{l}1 \\
0\end{array}$ & $\begin{array}{l}0 \\
0\end{array}$ & $\begin{array}{l}0 \\
0\end{array}$ & $\begin{array}{l}0 \\
0\end{array}$ & $\begin{array}{l}0 \\
0\end{array}$ & $\begin{array}{l}0 \\
0\end{array}$ & $\begin{array}{l}0 \\
0\end{array}$ & $\begin{array}{l}0 \\
0\end{array}$ & $\begin{array}{l}0 \\
0\end{array}$ & $\begin{array}{l}0 \\
0\end{array}$ & $\begin{array}{l}0 \\
0\end{array}$ & $\begin{array}{l}0 \\
0\end{array}$ & $\begin{array}{l}0 \\
0\end{array}$ & 0 & 0 & 0 \\
\hline Eunotia paludosa Grunow [V] & $\begin{array}{l}0 \\
1\end{array}$ & $\begin{array}{l}0 \\
0\end{array}$ & $\begin{array}{l}0 \\
0\end{array}$ & $\begin{array}{l}0 \\
0\end{array}$ & $\begin{array}{l}0 \\
0\end{array}$ & $\begin{array}{l}0 \\
0\end{array}$ & $\begin{array}{l}0 \\
0\end{array}$ & $\begin{array}{l}0 \\
0\end{array}$ & $\begin{array}{l}0 \\
0\end{array}$ & $\begin{array}{l}0 \\
0\end{array}$ & $\begin{array}{l}0 \\
0\end{array}$ & $\begin{array}{l}0 \\
0\end{array}$ & $\begin{array}{l}0 \\
0\end{array}$ & $\begin{array}{l}0 \\
0\end{array}$ & $\begin{array}{l}0 \\
0\end{array}$ & $\begin{array}{l}0 \\
0\end{array}$ & $\begin{array}{l}0 \\
0\end{array}$ & $\begin{array}{l}0 \\
0\end{array}$ & 0 & 0 & 0 \\
\hline $\begin{array}{l}\text { Eunotia superbidens Lange- } \\
\text { Bertalot } \\
(55.4 \times 13.6 ; \mathrm{s}: 10.5 ; \mathrm{n}=1)\end{array}$ & $\begin{array}{l}0 \\
0\end{array}$ & $\begin{array}{l}0 \\
0\end{array}$ & $\begin{array}{l}0 \\
0\end{array}$ & $\begin{array}{l}0 \\
0\end{array}$ & $\begin{array}{l}0 \\
0\end{array}$ & $\begin{array}{l}0 \\
0\end{array}$ & $\begin{array}{l}0 \\
0\end{array}$ & $\begin{array}{l}0 \\
0\end{array}$ & $\begin{array}{l}0 \\
0\end{array}$ & $\begin{array}{l}0 \\
0\end{array}$ & $\begin{array}{l}0 \\
0\end{array}$ & $\begin{array}{l}0 \\
0\end{array}$ & $\begin{array}{l}0 \\
0\end{array}$ & $\begin{array}{l}0 \\
0\end{array}$ & $\begin{array}{l}0 \\
0\end{array}$ & $\begin{array}{l}0 \\
0\end{array}$ & $\begin{array}{l}0 \\
2\end{array}$ & $\begin{array}{l}0 \\
0\end{array}$ & 0 & 0 & 0 \\
\hline $\begin{array}{l}\text { Eunotia ursamaioris Lange- } \\
\text { Bertalot \& Nörpel-Schempp } \\
(32.3 \times 5.9 ; \mathrm{s}: 16 \mathrm{n}=1)\end{array}$ & $\begin{array}{l}0 \\
0\end{array}$ & $\begin{array}{l}0 \\
0\end{array}$ & $\begin{array}{l}0 \\
0\end{array}$ & $\begin{array}{l}0 \\
0\end{array}$ & $\begin{array}{l}0 \\
0\end{array}$ & $\begin{array}{l}1 \\
0\end{array}$ & $\begin{array}{l}0 \\
0\end{array}$ & $\begin{array}{l}0 \\
0\end{array}$ & $\begin{array}{l}0 \\
0\end{array}$ & $\begin{array}{l}0 \\
0\end{array}$ & $\begin{array}{l}0 \\
0\end{array}$ & $\begin{array}{l}0 \\
0\end{array}$ & $\begin{array}{l}0 \\
0\end{array}$ & $\begin{array}{l}0 \\
0\end{array}$ & $\begin{array}{l}0 \\
0\end{array}$ & $\begin{array}{l}0 \\
0\end{array}$ & $\begin{array}{l}0 \\
0\end{array}$ & $\begin{array}{l}0 \\
0\end{array}$ & 0 & 0 & 0 \\
\hline $\begin{array}{l}\text { Eunotia valida Hustedt [I] } \\
(37.4-54.5 \times 3.7-5.0 ; \mathrm{s}: 12-13 \text {; } \\
\mathrm{n}=2)\end{array}$ & $\begin{array}{l}0 \\
0\end{array}$ & $\begin{array}{l}0 \\
0\end{array}$ & $\begin{array}{l}0 \\
0\end{array}$ & $\begin{array}{l}0 \\
0\end{array}$ & $\begin{array}{l}0 \\
0\end{array}$ & $\begin{array}{l}0 \\
0\end{array}$ & $\begin{array}{l}0 \\
0\end{array}$ & $\begin{array}{l}0 \\
0\end{array}$ & $\begin{array}{l}0 \\
0\end{array}$ & $\begin{array}{l}0 \\
0\end{array}$ & $\begin{array}{l}0 \\
0\end{array}$ & $\begin{array}{l}0 \\
0\end{array}$ & $\begin{array}{l}0 \\
0\end{array}$ & $\begin{array}{l}0 \\
0\end{array}$ & $\begin{array}{l}0 \\
0\end{array}$ & $\begin{array}{l}0 \\
0\end{array}$ & $\begin{array}{l}0 \\
4\end{array}$ & $\begin{array}{l}0 \\
0\end{array}$ & 0 & 0 & 0 \\
\hline $\begin{array}{l}\text { Eunotia cf. dorofeyukiae } \\
\text { Lange-Bertalot \& Kulikovskiy } \\
\text { (26.2-40.4×5.8-6.5; s: 13-15 } \\
\text { (18 SEM); a: 34-36; n=7) }\end{array}$ & $\begin{array}{l}0 \\
0\end{array}$ & $\begin{array}{l}0 \\
0\end{array}$ & $\begin{array}{l}0 \\
0\end{array}$ & $\begin{array}{l}0 \\
0\end{array}$ & $\begin{array}{l}0 \\
0\end{array}$ & $\begin{array}{l}0 \\
0\end{array}$ & $\begin{array}{l}0 \\
0\end{array}$ & $\begin{array}{l}0 \\
0\end{array}$ & $\begin{array}{l}0 \\
0\end{array}$ & $\begin{array}{l}0 \\
0\end{array}$ & $\begin{array}{l}0 \\
0\end{array}$ & $\begin{array}{l}0 \\
0\end{array}$ & $\begin{array}{l}0 \\
0\end{array}$ & $\begin{array}{l}0 \\
1\end{array}$ & $\begin{array}{l}0 \\
0\end{array}$ & $\begin{array}{l}0 \\
0\end{array}$ & $\begin{array}{l}5 \\
3\end{array}$ & $\begin{array}{c}15 \\
8\end{array}$ & 0 & 0 & 0 \\
\hline $\begin{array}{l}\text { Eunotia cf. glacialifalsa Lange- } \\
\text { Bertalot } \\
(93.8 \times 4.4 ; \mathrm{s}: 11 ; \mathrm{n}=1)\end{array}$ & $\begin{array}{l}0 \\
0\end{array}$ & $\begin{array}{l}0 \\
0\end{array}$ & $\begin{array}{l}0 \\
0\end{array}$ & $\begin{array}{l}0 \\
0\end{array}$ & $\begin{array}{l}0 \\
0\end{array}$ & $\begin{array}{l}0 \\
0\end{array}$ & $\begin{array}{l}0 \\
0\end{array}$ & $\begin{array}{l}0 \\
0\end{array}$ & $\begin{array}{l}0 \\
0\end{array}$ & $\begin{array}{l}0 \\
0\end{array}$ & $\begin{array}{l}0 \\
0\end{array}$ & $\begin{array}{l}0 \\
0\end{array}$ & $\begin{array}{l}0 \\
0\end{array}$ & $\begin{array}{l}0 \\
0\end{array}$ & $\begin{array}{l}0 \\
0\end{array}$ & $\begin{array}{l}0 \\
0\end{array}$ & $\begin{array}{l}0 \\
0\end{array}$ & $\begin{array}{l}0 \\
1\end{array}$ & 0 & 0 & 0 \\
\hline $\begin{array}{l}\text { Eunotia cf. groenlandica } \\
\text { (Grunow) Nörpel-Schempp \& } \\
\text { Lange-Bertalot } \\
(26.6-47.7 \times 3.8-4.2 ; \mathrm{s:} 13-15 ; \\
\text { a: } 35 ; \mathrm{n}=2)\end{array}$ & $\begin{array}{l}0 \\
0\end{array}$ & $\begin{array}{l}0 \\
0\end{array}$ & $\begin{array}{l}0 \\
0\end{array}$ & $\begin{array}{l}0 \\
0\end{array}$ & $\begin{array}{l}0 \\
0\end{array}$ & $\begin{array}{l}0 \\
0\end{array}$ & $\begin{array}{l}0 \\
0\end{array}$ & $\begin{array}{l}0 \\
0\end{array}$ & $\begin{array}{l}0 \\
0\end{array}$ & $\begin{array}{l}0 \\
0\end{array}$ & $\begin{array}{l}0 \\
0\end{array}$ & $\begin{array}{l}0 \\
0\end{array}$ & $\begin{array}{l}0 \\
0\end{array}$ & $\begin{array}{l}0 \\
0\end{array}$ & $\begin{array}{l}0 \\
0\end{array}$ & $\begin{array}{l}0 \\
0\end{array}$ & $\begin{array}{l}0 \\
2\end{array}$ & $\begin{array}{l}0 \\
0\end{array}$ & 0 & 0 & 0 \\
\hline $\begin{array}{l}\text { Pinnularia appendiculata (C. } \\
\text { Agardh) Schaarschmidt }\end{array}$ & $\begin{array}{l}0 \\
0\end{array}$ & $\begin{array}{l}0 \\
0 \\
\end{array}$ & $\begin{array}{l}0 \\
0\end{array}$ & $\begin{array}{l}0 \\
0\end{array}$ & 0 & $\begin{array}{l}0 \\
0\end{array}$ & $\begin{array}{l}0 \\
0 \\
\end{array}$ & $\begin{array}{l}0 \\
0\end{array}$ & $\begin{array}{l}0 \\
0\end{array}$ & $\begin{array}{l}0 \\
0\end{array}$ & $\begin{array}{l}1 \\
0\end{array}$ & $\begin{array}{l}0 \\
0\end{array}$ & $\begin{array}{l}0 \\
0\end{array}$ & $\begin{array}{l}0 \\
0\end{array}$ & $\begin{array}{l}0 \\
0\end{array}$ & $\begin{array}{l}0 \\
0\end{array}$ & $\begin{array}{l}0 \\
0\end{array}$ & $\begin{array}{l}0 \\
0\end{array}$ & 0 & 0 & 0 \\
\hline $\begin{array}{l}\text { Pinnularia borealis Ehrenberg } \\
(19.8-47.5 \times 5.6-11.0 \text {; s: } 5-7 \text {; } \\
n=31)\end{array}$ & $\begin{array}{c}20 \\
7\end{array}$ & $\begin{array}{c}24 \\
2\end{array}$ & $\begin{array}{c}16 \\
3\end{array}$ & $\begin{array}{l}5 \\
0\end{array}$ & $\begin{array}{l}6 \\
0\end{array}$ & $\begin{array}{l}0 \\
0\end{array}$ & $\begin{array}{l}19 \\
11\end{array}$ & $\begin{array}{l}54 \\
16\end{array}$ & $\begin{array}{l}12 \\
21\end{array}$ & $\begin{array}{l}23 \\
61\end{array}$ & $\begin{array}{c}43 \\
0\end{array}$ & $\begin{array}{c}0 \\
29\end{array}$ & $\begin{array}{l}50 \\
15\end{array}$ & $\begin{array}{c}7 \\
19\end{array}$ & $\begin{array}{c}0 \\
83\end{array}$ & $\begin{array}{l}1 \\
7\end{array}$ & $\begin{array}{l}12 \\
24\end{array}$ & $\begin{array}{c}13 \\
1\end{array}$ & 76 & 59 & 47 \\
\hline $\begin{array}{l}\text { Pinnularia borealis var. } \\
\text { sublinearis Krammer } \\
(26.6 \times 5.9 ; \mathrm{s}: 6 ; n=1)\end{array}$ & $\begin{array}{l}1 \\
0\end{array}$ & $\begin{array}{l}0 \\
0\end{array}$ & $\begin{array}{l}0 \\
0\end{array}$ & $\begin{array}{c}0 \\
37\end{array}$ & $\begin{array}{l}1 \\
7\end{array}$ & $\begin{array}{c}0 \\
11\end{array}$ & $\begin{array}{l}0 \\
0\end{array}$ & $\begin{array}{l}0 \\
0\end{array}$ & $\begin{array}{l}0 \\
0\end{array}$ & $\begin{array}{l}0 \\
0\end{array}$ & $\begin{array}{l}0 \\
0\end{array}$ & $\begin{array}{l}0 \\
0\end{array}$ & $\begin{array}{l}8 \\
0\end{array}$ & $\begin{array}{l}0 \\
0\end{array}$ & $\begin{array}{l}0 \\
0\end{array}$ & $\begin{array}{l}0 \\
0\end{array}$ & $\begin{array}{l}0 \\
0\end{array}$ & $\begin{array}{l}0 \\
0\end{array}$ & 0 & 2 & 0 \\
\hline $\begin{array}{l}\text { Pinnularia cuneola Reichardt } \\
(15.8-26 \times 4.8-6.8 ; \text { s: 13-14; } \\
\mathrm{n}=4)\end{array}$ & $\begin{array}{l}0 \\
0\end{array}$ & $\begin{array}{l}0 \\
0\end{array}$ & $\begin{array}{l}0 \\
0\end{array}$ & 0 & $\begin{array}{l}0 \\
0\end{array}$ & $\begin{array}{l}0 \\
0\end{array}$ & $\begin{array}{l}0 \\
0\end{array}$ & $\begin{array}{l}0 \\
0\end{array}$ & $\begin{array}{l}0 \\
0\end{array}$ & $\begin{array}{l}0 \\
0\end{array}$ & $\begin{array}{l}0 \\
0\end{array}$ & $\begin{array}{l}9 \\
0\end{array}$ & $\begin{array}{l}0 \\
0\end{array}$ & $\begin{array}{l}0 \\
0\end{array}$ & $\begin{array}{l}0 \\
0\end{array}$ & $\begin{array}{l}0 \\
0\end{array}$ & $\begin{array}{l}0 \\
0\end{array}$ & $\begin{array}{l}0 \\
0\end{array}$ & 0 & 0 & 6 \\
\hline $\begin{array}{l}\text { Pinnularia frequentis Krammer } \\
(43.6-59.7 \times 8-9.7 \text {; s: } 10-11 \text {; } \\
n=9)\end{array}$ & $\begin{array}{l}0 \\
0\end{array}$ & $\begin{array}{l}0 \\
0\end{array}$ & $\begin{array}{l}0 \\
0\end{array}$ & 0 & $\begin{array}{c}0 \\
11\end{array}$ & $\begin{array}{l}0 \\
9\end{array}$ & $\begin{array}{l}0 \\
0\end{array}$ & $\begin{array}{l}0 \\
0\end{array}$ & $\begin{array}{l}0 \\
0\end{array}$ & $\begin{array}{l}0 \\
0\end{array}$ & $\begin{array}{l}1 \\
0\end{array}$ & $\begin{array}{l}0 \\
0\end{array}$ & $\begin{array}{l}0 \\
0\end{array}$ & $\begin{array}{l}1 \\
0\end{array}$ & $\begin{array}{l}0 \\
2\end{array}$ & $\begin{array}{l}0 \\
0\end{array}$ & $\begin{array}{l}5 \\
0\end{array}$ & 0 & 0 & 3 & 0 \\
\hline $\begin{array}{l}\text { Pinnularia grunowii Krammer } \\
(36.7 \times 7.7 ; \mathrm{s}: 12 ; \mathrm{n}=1)\end{array}$ & $\begin{array}{l}0 \\
0 \\
\end{array}$ & $\begin{array}{l}0 \\
0\end{array}$ & $\begin{array}{l}0 \\
0\end{array}$ & 0 & 0 & $\begin{array}{l}0 \\
0\end{array}$ & $\begin{array}{l}0 \\
0 \\
\end{array}$ & $\begin{array}{l}0 \\
0 \\
\end{array}$ & $\begin{array}{l}0 \\
0 \\
\end{array}$ & $\begin{array}{l}0 \\
0 \\
\end{array}$ & $\begin{array}{l}0 \\
0 \\
\end{array}$ & $\begin{array}{l}0 \\
0 \\
\end{array}$ & $\begin{array}{l}0 \\
0 \\
\end{array}$ & $\begin{array}{l}0 \\
0 \\
\end{array}$ & $\begin{array}{l}0 \\
0 \\
\end{array}$ & $\begin{array}{l}0 \\
0 \\
\end{array}$ & $\begin{array}{l}0 \\
0 \\
\end{array}$ & $\begin{array}{l}0 \\
9 \\
\end{array}$ & 0 & 0 & 0 \\
\hline $\begin{array}{l}\text { Pinnularia intermedia } \\
\text { (Lagerstedt) Cleve } \\
(22.1 \times 5.6 ; \mathrm{s}: 8 \mathrm{n}=1)\end{array}$ & $\begin{array}{l}0 \\
0\end{array}$ & $\begin{array}{l}0 \\
0\end{array}$ & $\begin{array}{l}0 \\
0\end{array}$ & $\begin{array}{l}0 \\
0\end{array}$ & $\begin{array}{l}0 \\
0\end{array}$ & $\begin{array}{l}0 \\
0\end{array}$ & $\begin{array}{l}0 \\
0\end{array}$ & $\begin{array}{l}0 \\
0\end{array}$ & $\begin{array}{l}0 \\
0\end{array}$ & $\begin{array}{l}0 \\
0\end{array}$ & $\begin{array}{l}0 \\
0\end{array}$ & $\begin{array}{l}0 \\
0\end{array}$ & $\begin{array}{l}0 \\
0\end{array}$ & $\begin{array}{l}0 \\
0\end{array}$ & $\begin{array}{l}0 \\
0\end{array}$ & $\begin{array}{l}0 \\
0\end{array}$ & $\begin{array}{l}0 \\
0\end{array}$ & $\begin{array}{l}0 \\
3\end{array}$ & 0 & 0 & 0 \\
\hline $\begin{array}{l}\text { Pinnularia isselana Krammer } \\
(30.4-56.8 \times 6.3-10.3 ; \mathrm{s}: 10-13 \text {; } \\
\mathrm{n}=10)\end{array}$ & $\begin{array}{l}1 \\
0\end{array}$ & $\begin{array}{l}0 \\
0\end{array}$ & $\begin{array}{l}0 \\
0\end{array}$ & $\begin{array}{l}0 \\
0\end{array}$ & $\begin{array}{l}2 \\
0\end{array}$ & $\begin{array}{l}1 \\
3\end{array}$ & $\begin{array}{l}0 \\
0\end{array}$ & $\begin{array}{l}1 \\
0\end{array}$ & $\begin{array}{l}0 \\
0\end{array}$ & $\begin{array}{l}0 \\
0\end{array}$ & $\begin{array}{l}0 \\
0\end{array}$ & $\begin{array}{l}0 \\
0\end{array}$ & $\begin{array}{l}0 \\
0\end{array}$ & $\begin{array}{l}2 \\
1\end{array}$ & $\begin{array}{l}0 \\
0\end{array}$ & $\begin{array}{l}0 \\
0\end{array}$ & $\begin{array}{l}0 \\
0\end{array}$ & $\begin{array}{c}1 \\
28\end{array}$ & 0 & 0 & 1 \\
\hline
\end{tabular}


Table 2. cont.

\begin{tabular}{|c|c|c|c|c|c|c|c|c|c|c|c|c|c|c|c|c|c|c|c|c|c|}
\hline Year & & 2013 & & & 2014 & & & 2015 & & & 2016 & & & 2017 & & & 2018 & & & 2019 & \\
\hline $\begin{array}{l}\text { Pinnularia marchica } \\
\text { Schönfelder } \\
(23.3-28.6 \times 4.7-5.3 ; \text { s: } 12-14 ; \\
n=4)\end{array}$ & $\begin{array}{l}0 \\
0\end{array}$ & $\begin{array}{l}0 \\
0\end{array}$ & $\begin{array}{l}0 \\
0\end{array}$ & $\begin{array}{l}0 \\
0\end{array}$ & $\begin{array}{l}0 \\
0\end{array}$ & $\begin{array}{l}0 \\
0\end{array}$ & $\begin{array}{l}0 \\
0\end{array}$ & $\begin{array}{l}0 \\
0\end{array}$ & $\begin{array}{l}0 \\
0\end{array}$ & $\begin{array}{l}0 \\
0\end{array}$ & $\begin{array}{l}0 \\
0\end{array}$ & $\begin{array}{l}0 \\
0\end{array}$ & $\begin{array}{l}0 \\
0\end{array}$ & $\begin{array}{l}0 \\
0\end{array}$ & $\begin{array}{l}0 \\
0\end{array}$ & $\begin{array}{l}0 \\
0\end{array}$ & $\begin{array}{l}0 \\
5\end{array}$ & $\begin{array}{c}0 \\
20\end{array}$ & 0 & 0 & 0 \\
\hline $\begin{array}{l}\text { g) Cleve [V] } \\
\text { s: } 11 ; n=1)\end{array}$ & $\begin{array}{l}0 \\
0\end{array}$ & $\begin{array}{l}0 \\
0\end{array}$ & $\begin{array}{l}0 \\
0\end{array}$ & $\begin{array}{l}0 \\
0\end{array}$ & $\begin{array}{l}0 \\
0\end{array}$ & $\begin{array}{l}0 \\
0\end{array}$ & $\begin{array}{l}0 \\
0\end{array}$ & $\begin{array}{l}0 \\
0\end{array}$ & $\begin{array}{l}0 \\
0\end{array}$ & $\begin{array}{l}0 \\
0\end{array}$ & $\begin{array}{l}0 \\
0\end{array}$ & $\begin{array}{l}0 \\
0\end{array}$ & $\begin{array}{l}0 \\
0\end{array}$ & $\begin{array}{l}0 \\
0\end{array}$ & $\begin{array}{l}0 \\
0\end{array}$ & $\begin{array}{l}0 \\
0\end{array}$ & $\begin{array}{l}0 \\
0\end{array}$ & $\begin{array}{l}0 \\
1\end{array}$ & 0 & 0 & 0 \\
\hline $\begin{array}{l}\text { Pinnularia nodosa (Ehrenberg) } \\
\text { Smith [E] } \\
(32.4-66 \times 5.8-8.5 ; \text { s: } 10-12 ; \\
n=5)\end{array}$ & $\begin{array}{l}0 \\
0\end{array}$ & $\begin{array}{l}0 \\
0\end{array}$ & $\begin{array}{l}0 \\
0\end{array}$ & $\begin{array}{l}0 \\
0\end{array}$ & $\begin{array}{l}0 \\
0\end{array}$ & $\begin{array}{l}0 \\
0\end{array}$ & $\begin{array}{l}0 \\
0\end{array}$ & $\begin{array}{l}0 \\
0\end{array}$ & $\begin{array}{l}0 \\
0\end{array}$ & $\begin{array}{l}0 \\
0\end{array}$ & $\begin{array}{l}0 \\
0\end{array}$ & $\begin{array}{l}0 \\
0\end{array}$ & $\begin{array}{l}0 \\
0\end{array}$ & $\begin{array}{l}0 \\
0\end{array}$ & $\begin{array}{l}0 \\
0\end{array}$ & $\begin{array}{l}0 \\
0\end{array}$ & $\begin{array}{l}0 \\
3\end{array}$ & $\begin{array}{l}0 \\
4\end{array}$ & 0 & 0 & 0 \\
\hline $\begin{array}{l}\text { laria obscura Krasske } \\
-26.6 \times 3.5-4.8 \text {; s: 10-16; }\end{array}$ & $\begin{array}{c}24 \\
8\end{array}$ & $\begin{array}{c}23 \\
0\end{array}$ & $\begin{array}{c}64 \\
5\end{array}$ & $\begin{array}{l}11 \\
28\end{array}$ & $\begin{array}{c}2 \\
11\end{array}$ & $\begin{array}{c}7 \\
10\end{array}$ & $\begin{array}{l}8 \\
7\end{array}$ & $\begin{array}{l}3 \\
3\end{array}$ & $\begin{array}{c}3 \\
10\end{array}$ & $\begin{array}{l}1 \\
7\end{array}$ & 3 & $\begin{array}{l}4 \\
6\end{array}$ & $\begin{array}{c}9 \\
50\end{array}$ & $\begin{array}{l}10 \\
14\end{array}$ & $\begin{array}{c}8 \\
79\end{array}$ & $\begin{array}{l}7 \\
1\end{array}$ & $\begin{array}{l}2 \\
5\end{array}$ & $\begin{array}{c}0 \\
56\end{array}$ & 47 & 13 & 27 \\
\hline $\begin{array}{l}\text { Pinnularia schoenfelderii } \\
\text { (Hustedt) Krammer [E] } \\
(18.4-27.5 \times 3.6-5.2 ; \text { s: } 14-17 \text {; } \\
n=6)\end{array}$ & $\begin{array}{l}1 \\
0\end{array}$ & $\begin{array}{l}0 \\
0\end{array}$ & $\begin{array}{l}0 \\
0\end{array}$ & $\begin{array}{l}4 \\
1\end{array}$ & $\begin{array}{l}1 \\
3\end{array}$ & $\begin{array}{c}0 \\
11\end{array}$ & $\begin{array}{c}0 \\
10\end{array}$ & $\begin{array}{l}0 \\
1\end{array}$ & $\begin{array}{l}0 \\
1\end{array}$ & $\begin{array}{l}0 \\
0\end{array}$ & $\begin{array}{l}6 \\
0\end{array}$ & $\begin{array}{l}0 \\
0\end{array}$ & $\begin{array}{l}0 \\
0\end{array}$ & $\begin{array}{l}0 \\
0\end{array}$ & $\begin{array}{l}0 \\
0\end{array}$ & $\begin{array}{l}0 \\
0\end{array}$ & $\begin{array}{l}0 \\
2\end{array}$ & $\begin{array}{l}0 \\
0\end{array}$ & 0 & 0 & 0 \\
\hline $\begin{array}{l}\text { Pinnularia silvatica Petersen } \\
(17.4-19.4 \times 3.5-3.8 \text {; s: } 20-24 \text {; } \\
n=6)\end{array}$ & $\begin{array}{l}0 \\
0\end{array}$ & $\begin{array}{l}0 \\
0\end{array}$ & $\begin{array}{l}0 \\
0\end{array}$ & $\begin{array}{l}0 \\
0\end{array}$ & $\begin{array}{l}0 \\
0\end{array}$ & $\begin{array}{l}0 \\
0\end{array}$ & $\begin{array}{l}0 \\
0\end{array}$ & $\begin{array}{l}0 \\
0\end{array}$ & $\begin{array}{l}0 \\
0\end{array}$ & $\begin{array}{l}0 \\
0\end{array}$ & 0 & $\begin{array}{l}0 \\
0\end{array}$ & $\begin{array}{l}0 \\
1\end{array}$ & $\begin{array}{l}0 \\
0\end{array}$ & $\begin{array}{l}0 \\
0\end{array}$ & $\begin{array}{l}0 \\
0\end{array}$ & $\begin{array}{l}0 \\
1\end{array}$ & 0 & 0 & 0 & 0 \\
\hline $\begin{array}{l}\text { Pinnularia sinistra Krammer } \\
(9.8-40 \times 3.8-5.0 ; \mathrm{s}: 10-14 \mathrm{p} ; \\
\mathrm{n}=8)\end{array}$ & $\begin{array}{l}1 \\
0\end{array}$ & $\begin{array}{l}0 \\
0\end{array}$ & $\begin{array}{l}0 \\
0\end{array}$ & $\begin{array}{l}0 \\
1\end{array}$ & $\begin{array}{l}0 \\
0\end{array}$ & $\begin{array}{l}0 \\
0\end{array}$ & $\begin{array}{l}2 \\
0\end{array}$ & $\begin{array}{l}1 \\
0\end{array}$ & $\begin{array}{l}0 \\
0\end{array}$ & $\begin{array}{l}0 \\
1\end{array}$ & 0 & $\begin{array}{l}0 \\
0\end{array}$ & $\begin{array}{l}0 \\
1\end{array}$ & $\begin{array}{l}2 \\
9\end{array}$ & $\begin{array}{l}2 \\
6\end{array}$ & $\begin{array}{l}0 \\
0\end{array}$ & $\begin{array}{l}0 \\
7\end{array}$ & $\begin{array}{l}0 \\
7\end{array}$ & 2 & 5 & 0 \\
\hline $\begin{array}{l}\text { Pinnularia viridiformis Krammer } \\
\text { [E] } \\
(88.8-117.6 \times 13.8-15.8 ; \mathrm{s}: \\
8-9 ; \mathrm{n}=2)\end{array}$ & $\begin{array}{l}0 \\
0\end{array}$ & $\begin{array}{l}0 \\
0\end{array}$ & $\begin{array}{l}0 \\
0\end{array}$ & $\begin{array}{l}0 \\
0\end{array}$ & $\begin{array}{l}0 \\
0\end{array}$ & $\begin{array}{l}0 \\
0\end{array}$ & $\begin{array}{l}0 \\
0\end{array}$ & $\begin{array}{l}0 \\
0\end{array}$ & $\begin{array}{l}0 \\
0\end{array}$ & $\begin{array}{l}0 \\
0\end{array}$ & $\begin{array}{l}0 \\
0\end{array}$ & $\begin{array}{l}0 \\
0\end{array}$ & $\begin{array}{l}0 \\
0\end{array}$ & $\begin{array}{l}0 \\
0\end{array}$ & $\begin{array}{l}0 \\
0\end{array}$ & $\begin{array}{l}0 \\
0\end{array}$ & $\begin{array}{l}0 \\
3\end{array}$ & 0 & 0 & 0 & 0 \\
\hline $\begin{array}{l}\text { aria viridis (Nitzsch) } \\
\text { erg } \\
\times 22.1 ; \mathrm{s}: 7 ; \mathrm{n}=1 \text { ) }\end{array}$ & $\begin{array}{l}0 \\
0\end{array}$ & $\begin{array}{l}0 \\
0\end{array}$ & $\begin{array}{l}0 \\
0\end{array}$ & $\begin{array}{l}0 \\
0\end{array}$ & $\begin{array}{l}0 \\
0\end{array}$ & $\begin{array}{l}0 \\
0\end{array}$ & $\begin{array}{l}0 \\
0\end{array}$ & $\begin{array}{l}0 \\
0\end{array}$ & $\begin{array}{l}0 \\
0\end{array}$ & $\begin{array}{l}0 \\
0\end{array}$ & $\begin{array}{l}0 \\
0\end{array}$ & $\begin{array}{l}0 \\
0\end{array}$ & $\begin{array}{l}0 \\
0\end{array}$ & $\begin{array}{l}0 \\
0\end{array}$ & $\begin{array}{l}0 \\
0\end{array}$ & $\begin{array}{l}0 \\
0\end{array}$ & $\begin{array}{l}0 \\
1\end{array}$ & 0 & 0 & 0 & 0 \\
\hline $\begin{array}{l}\text { Pinnularia cf. rabenhorstii var. } \\
\text { franconica Krammer } \\
(43 \times 11 ; \mathrm{s}: 5 ; \mathrm{n}=1)\end{array}$ & $\begin{array}{l}0 \\
0\end{array}$ & $\begin{array}{l}0 \\
0\end{array}$ & $\begin{array}{l}0 \\
0\end{array}$ & $\begin{array}{l}0 \\
0\end{array}$ & $\begin{array}{l}0 \\
0\end{array}$ & $\begin{array}{l}0 \\
0\end{array}$ & $\begin{array}{l}0 \\
0\end{array}$ & $\begin{array}{l}0 \\
0\end{array}$ & $\begin{array}{l}0 \\
0\end{array}$ & $\begin{array}{l}0 \\
0\end{array}$ & $\begin{array}{l}0 \\
0\end{array}$ & $\begin{array}{l}0 \\
0\end{array}$ & $\begin{array}{l}0 \\
0\end{array}$ & $\begin{array}{l}0 \\
0\end{array}$ & $\begin{array}{l}0 \\
0\end{array}$ & $\begin{array}{l}0 \\
0\end{array}$ & $\begin{array}{l}0 \\
0\end{array}$ & $\begin{array}{l}0 \\
0\end{array}$ & 0 & 0 & 2 \\
\hline
\end{tabular}

Categories of threats: E - Endangered, V - Vulnerable, I - Indeterminate; $n$ - means the number of specimens measured; Numbers in black represent the number of taxa in spring, while in red - in autumn.

The species of the Eunotia genus occurred individually in most sites, none of them dominated in the diatoms assemblage. Only E. curtagrunowii Nörpel-Schempp \& Lange-Bertalot (Fig. 1: 8-11, Fig. 2: 51-52) was identified in a larger number of valves (8-14) in 2014 and in the years 2016-2018, always in second site (R2). In the spring of 2018, Eunotia cf. dorofeyukae LangeBertalot \& Kulikovskiy (15 valves) developed abundantly on the third site (R3), Table 2, Fig. 1: 3, Fig. 2: 53, 54.

The species of the Pinnularia genus were more abundant than species of the Eunotia genus. Two species - Pinnularia borelis Ehrenberg (Fig. 1: 33-37, Fig. 2: 44, Fig. 3: 53, 54) and Pinnularia obscura Krasske (Fig. 1: 21-23, Fig. 3: 69) - dominated in different research seasons, reaching more than 50 valves in the diatoms assemblage. Pinnulara obscura more often dominated in autumn and created the largest populations at R3 in 2017 (79 valves). Pinnularia borealis also occurred most frequently in autumn in the third study site (R3) in 2017 (83 valves), however, it was more often the dominant species in spring. Other species of the Pinnularia genus were usually found in the form of single cells, only Pinnularia borealis var. sublinearis Krammer, Pinnularia isselana Krammer (Fig. 1: 27, 28, Fig 2: 41, Fig. 3: 57,60) and Pinnularia marchica Schönfelder (Fig. 1: 13,26, Fig. 2:46, Fig. 3: 67, 68) were found in individual research seasons in larger numbers, i.e. more than 20 valves in the assemblage (Table 2).

Some diatoms, e.g. Eunotia curtagrunowii, Pinnularia borealis, P. cuneola Reichardt (Fig. 1: 20, Fig. 2: 47 or P. sinistra Krammer (Fig. 1: 17, 18, Fig. 2: 43, Fig. 3: 65, 66), had smaller dimensions (both in length and width), compared to the literature data. Others - Eunotia cf. dorofeyukae and Pinnularia schoenfelderi (Hustedt) Krammer (Fig. 1: 12, 14, 15) 

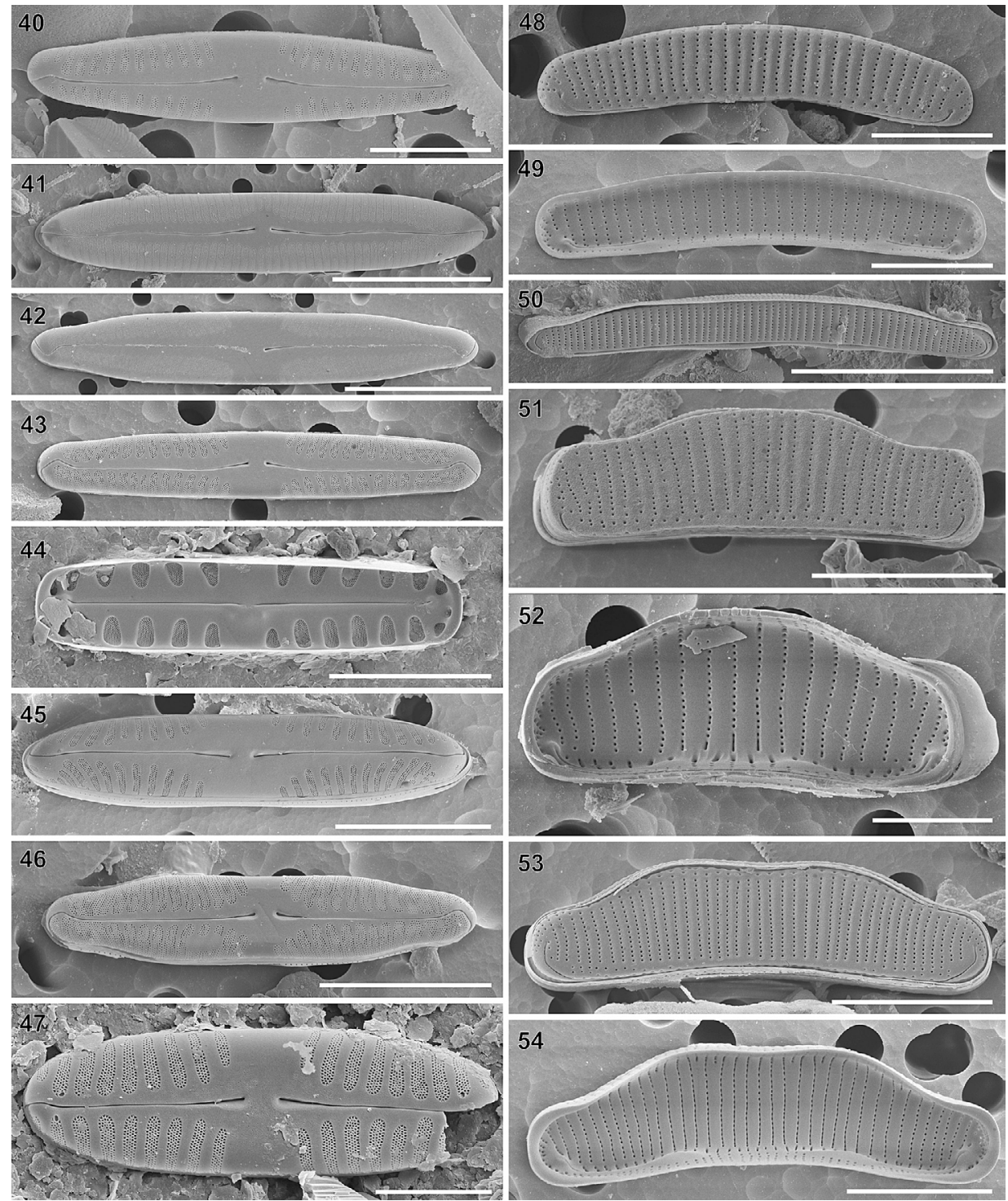

Figure 2. Selected diatom species designated in analyzed materials (SEM - microphotographs): 40 - Pinnularia cf. silvatica (scale bar $=5 \mu \mathrm{m}$ ), $41-P$. isselana (scale bar $=10 \mu \mathrm{m}$ ), $42-P$. microstauron (scale bar $=20 \mu \mathrm{m}$ ), $43-P$. sinistra (scale bar $=10 \mu \mathrm{m}), 44-P$. borealis (scale bar $=10 \mu \mathrm{m}), 45-P$. obscura $($ scale bar $=10 \mu \mathrm{m})$,

$46-P$. marchica (scale bar $=10 \mu \mathrm{m}), 47-P$. cuneola (scale bar $=5 \mu \mathrm{m}), 48,49-$ Eunotia botuliformis

(scale bar $=5 \mu \mathrm{m}$ ), $50-$ Eunotia cf. groenlandica (scale bar $=20 \mu \mathrm{m}$ ), 51, $52-$ E. curtagrunowii

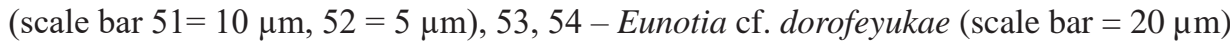

- were often much narrower compared to the literature.

The research conducted in the years 20132019 on the diversity of the soil diatom communities in the Ryjak stream valley showed the occurrence of 8 taxa inscribed in the Red List of the algae in Poland (Siemińska et al. 2006) in various categories of threat. Half of them are included in the endangered category (E): Eunotia botuliformis F. Wild, Nörpel \& Lange-Bert. (Fig. 1: 7, Fig. 2: 48, 49), Pinnularia nodosa (Ehrenberg) Smith (Fig. 1: 31, 32, Fig. 3: 58, 59), P. schoenfelderii and P. viridiformis Krammer (Fig. 1: 38, Fig. 3: 61, 62). The others are diatoms from the Vulnerable (V) category: Eunotia paludosa Grunow and Pinnularia microstauron (Ehrenberg) Cleve (Fig. 2: 42, Fig. 3: $63,64)$, and from the Indeterminate category [I]: Eunotia meisteri Hustedt and E. valida Hustedt (Table 2) - Fig. 1: 4.

In the spring of 2016 and 2019, Pinnularia cuneola appeared in the third site (R3) (6-9 valves in the preparation, Table 2). So far, it has not been reported from soils, and the site in the Low Beskids is still the second place of occurrence in Poland.

In order to investigate the impact of $\mathrm{pH}$ and habitat moisture on the differentiation of 

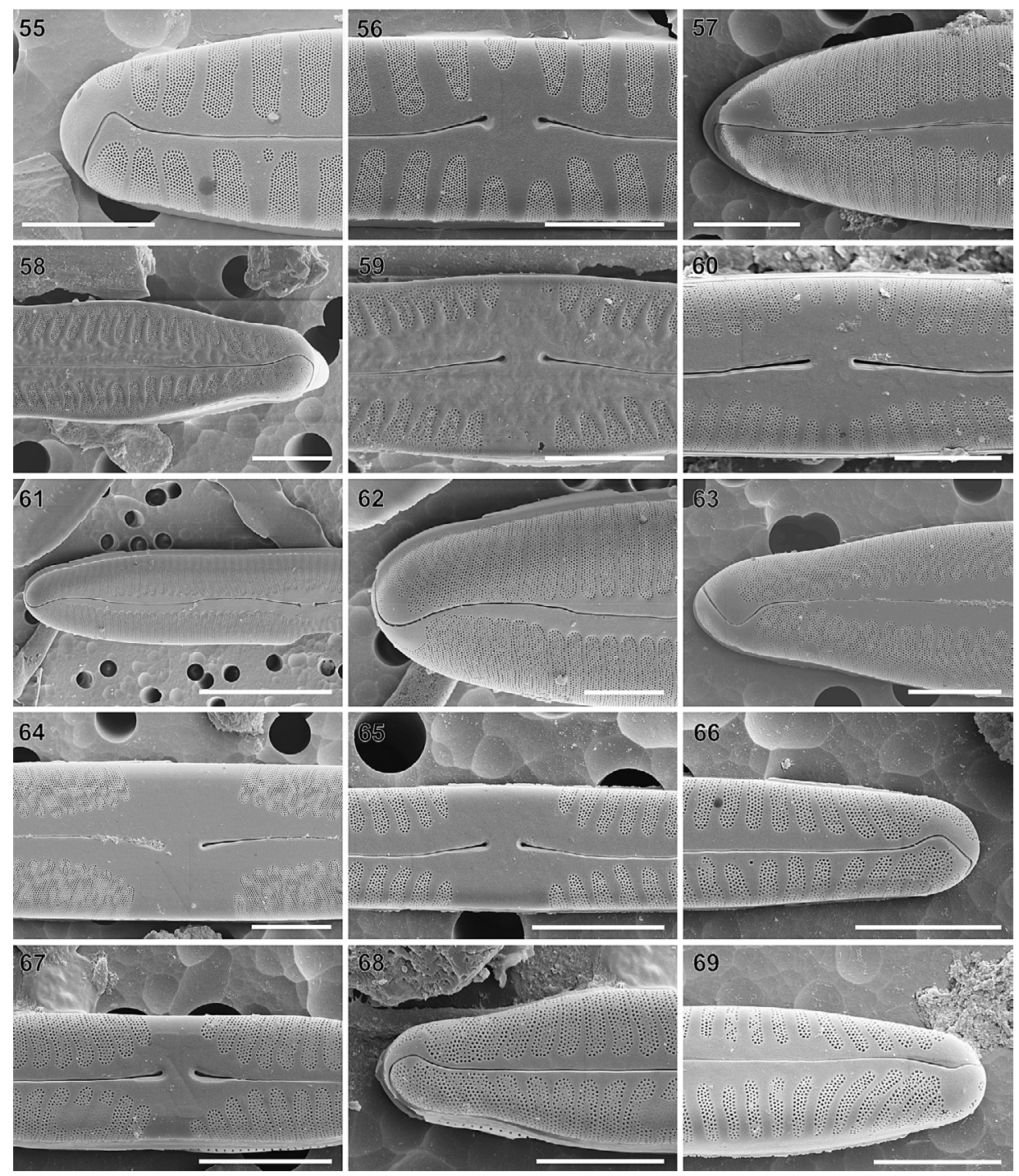

Figure 3. Selected diatom species designated in analyzed materials (SEM - microphotographs) - details of valve structures: 55, 56 - Pinnularia borealis, 57, 60 - P. isselana, 58, 59 - P. nodosa, 61, 62 - P. viridiformis,

63, 64 - P. microstauron, 65, 66 - P. sinistra, 67, 68 - P. marchica, 69 - P. obscura.

Scale bar $=5 \mu \mathrm{m}$, but $68,69=4 \mu \mathrm{m}$

diatom communities, canonical analysis (CCA) was performed. These variables are statistically significant $(\mathrm{p}<0.05)$. The analysis did not reveal high statistical significance (for the $\mathrm{pH}$ it explains 6\% variability, for humidity: $4.7 \%$ ).

The relationships between the environmental parameters and the diatom communities at individual sites are shown in Figure 4. The analysis shows that the communities were more affected by humidity in 2016-2018 in most seasons. It was also associated with greater development of taxa such as Pinnularia borealis and Pinnularia obscura in these years (Table 2), especially in autumn. This is also confirmed by the level of soil moisture, which in these seasons oscillated between 32.1 and $49.2 \%$ in spring and between 49.0 and $61.9 \%$ in autumn
(Table 1). Similar values were also recorded at individual sites in 2014 and 2015, but no increase in the number of taxa was observed, including the most numerous ones i.e. Pinnularia borealis and P. obscura.

\section{DISCUSSION}

The soils at the studied sites in the Magura National Park and the buffer zone were usually characterized by a high level of moisture, especially in autumn. The lowest moisture content was found in soils in the spring of 2015 and the autumn of 2013. In the first case, the material was collected in late spring, when there was no rainfall for a long time, while 2013 was designated 


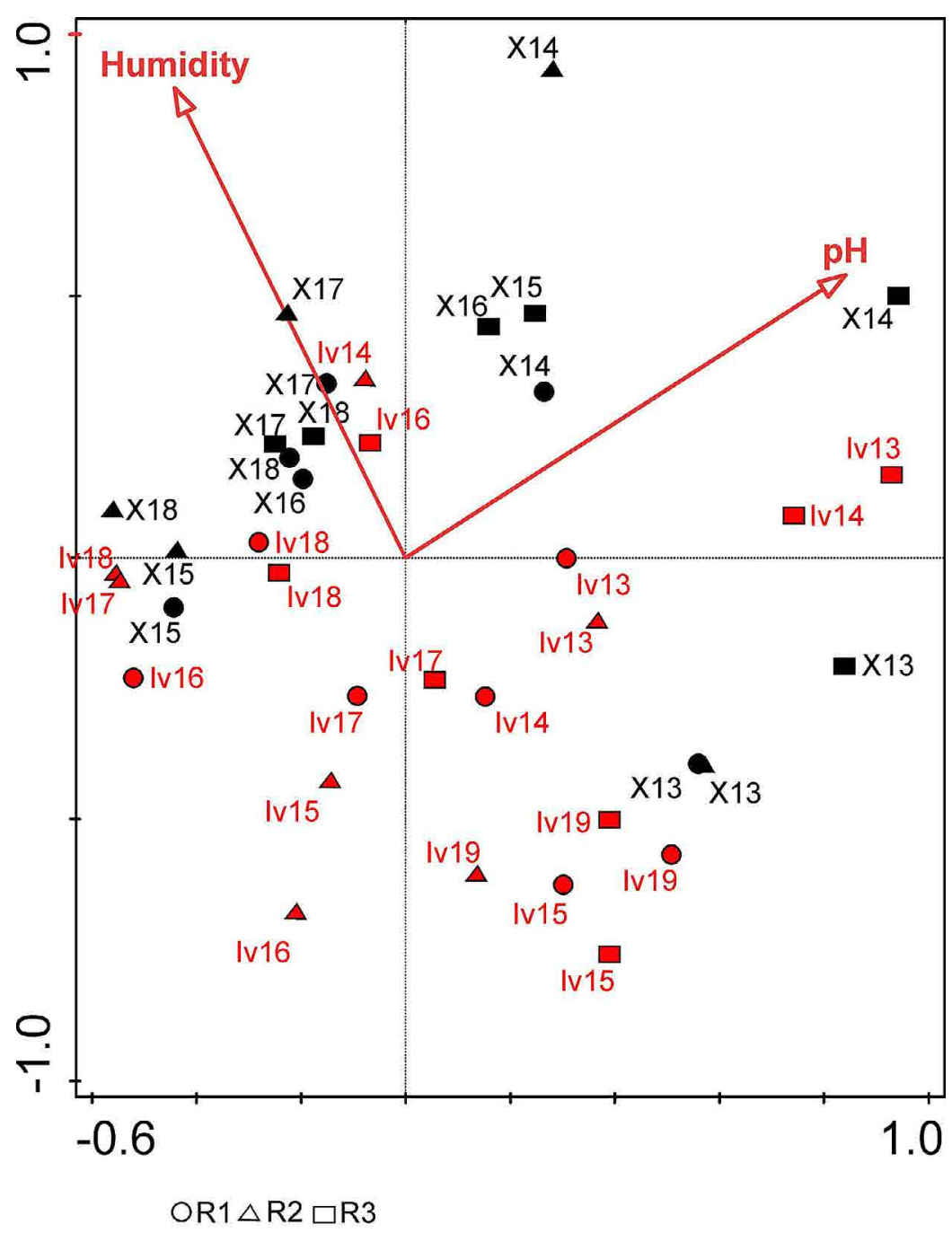

Figure 4. Diagram of canonical analysis (CCA) of the influence of $\mathrm{pH}$ and humidity of soil on the differentiation of diatom communities in individual sites (R1-3). Red color means spring season, while black - autumn

by prolonged drought in summer. Therefore, in these years the moisture level was the lowest during the seven-year study period. Many authors report that the level of moisture in the upper soil layer $(0-10 \mathrm{~cm})$ as well as the water supply and its availability for plants depend on the amount of precipitation (Krążel 1990, Pudełko et al. 1994, Ignaczak 1998, Włodek et al. 2007). Banach et al. (2015) reports the level of moisture content in brown soils, rendzinas, chernozems and rankers from the Lublin region in the range of $7-28 \%$. Very low humidity (1.9-6.0\%) was measured in sandy soils near Tarnów (Stanek-Tarkowska et al. 2016a), while the level of moisture on the clay soils near Rzeszów (Stanek-Tarkowska et al. 2017) was comparable to the results obtained from the Lublin region (11.3-33.8\%).

The soils at the sites in the Ryjak stream valley were acidic or slightly acidic in all the research seasons. The third site (R3) was characterized by slightly higher pH values, especially in 2013$2014(\mathrm{pH}>6)$ compared to the other two sites (R1 and R2). Similar $\mathrm{pH}$ values were measured for arable land and fallow land soils in other areas of south-eastern Poland. Clay soil under winter wheat and oats in Kosina near Rzeszów was characterized by an acidic pH (4.9-5.9), while sandy soil for spring wheat in Pogórska Wola near Tarnów was acidic or slightly acidic (pH: 4.8-6.4) (Stanek-Tarkowska et al. 2016, 2017). The lowest $\mathrm{pH}$ values (4.1-5.3) were recorded on the fallow soil in Pogórska Wola (Stanek-Tarkowska et al. 2015). Only dust soils under maize cultivation in Dąbrowa and Boguchwala near Rzeszów had a slightly acidic or neutral pH (5.8-7.2) (StanekTarkowska \& Noga 2012a, b). In Poland, almost $80 \%$ of arable land is acidified to a varying degree (very acidic $-29 \%$, acidic $-28 \%$ and slightly acidic $-24 \%$ ) and only about $20 \%$ are neutral or alkaline soils (Siebielec et al. 2017). 
The acidic $\mathrm{pH}$ of the soils in the Magura National Park and the buffer zone undoubtedly promotes the development of diatoms from the Eunotia and Pinnularia genera. Both genera prefer the environments associated with natural acidification, oligo- and dystrophic waters, usually with low electrolyte content, in which often (especially the species of the Eunotia genus) are indicators of very good water quality (Krammer 2000, Hofmann et al. 2011, Lange-Bertalot et al. 2011, 2017).

Most species of the Eunotia and Pinnularia genera usually developed individually on the studied soils, with the exception of Pinnularia borealis and P. obscura, which occurred in most of the research seasons and were often dominant. These are cosmopolitan species commonly found in various types of habitats, including soils, moist walls, rocks, among mosses and other periodically drying environments (Van Dam et al. 1994, Ettl \& Gärtner 1995, Krammer 2000, Lange-Bertalot et al. 2017). Both species often occur also in Poland (Siemińska \& Wołowski 2003), in southeastern Poland they are identified in most of the studied rivers and streams; however, they usually develop individually (Noga et al. 2014a, b). They formed more numerous populations on soils (most often on cultivated, but also fallow land) in south-eastern Poland and often dominated in the diatom communities (Stanek-Tarkowska \& Noga 2012a, b, Stanek-Tarkowska et al. 2015, 2016). Pinnularia borealis was also one of the main dominants among mosses growing on the bark of trees (Rybak et al. 2018).

Numerous development of Pinnularia borealis and P. obscura on soils in the Low Beskids in many research seasons, definitely confirms that they are typically soil species. Other diatoms of the Pinnularia genus usually occurred individually and the number of some species increased slightly on rare occasions (up to a dozen or several dozen cells on the site). The performed statistical analysis showed a certain correlation between the numerous development of these species at the studied sites and the increase in the level of moisture. Foets et al. (2020) indicated that diatoms have a number of features, including morphological ones that allow them to adapt to dynamic (daily, seasonal) changes in the environment, e.g. to the changes in moisture content. Therefore, in order to clarify this statistical relationship, further in-depth research on the ecology of these species is needed.
Species such as: Pinnularia frequentis, $P$. isselana or $P$. marchica were described for the first time in 2000, and their ecology (among others due to the rare occurrence) is still poorly understood (Krammer 2000). This research shows that they can also successfully grow on soils, especially those covered with mosses, but they do not form mass populations. Pinnularia isselana is known for oligotrophic waters with low electrolyte content (Krammer 2000, Hofmann et al. 2011). On the investigated soils, it occurred most frequently on the R3 site (28 valves) in 2018 at low $\mathrm{pH}$ and high moisture.

Many diatoms, both of the Pinnularia and Eunotia genera, reached smaller cell sizes in the material compared to the literature data. Especially Eunotia curtagrunowii often appeared in the form of very small specimens and many valves were shorter and narrower compared to the dimensions contained in the key (Lange-Bertalot et al. 2011). Eunotia curtagrunowii in the designated sites was one of the most frequently identified diatoms of its genus. Moreover, Holarctic is one of the most common species of the Eunotia genus. It prefers oligotrophic springs, marginal parts of peat bogs, dystrophic lakes and wet bryophytes. In all habitats, it occurs at low $\mathrm{pH}$ and low electrolyte content (Lange-Bertalot et al. 2011). On the studied soils, it was most abundant on the R2 site, which was covered with mosses in places. E. dorofeyu$k a e$, which in the studied material was designated as Eunotia cf. dorofeyukae, is characterized by a similar ecology due to high morphological variability and the presence of much narrower valves in most of the cells found $(5.8-7.8 \mu \mathrm{m}$ and not $7-8.5 \mu \mathrm{m}$ as reported by Lange-Bertalot et al. 2011). The species requires further investigation in the future, but the observations carried out so far indicate that in a soil environment with unstable conditions (e.g. frequent lack of water or even prolonged drought), many cells reach much smaller sizes and become clearly dwarfish.

In addition to cosmopolitan diatoms, widespread in various habitats, there are also rare or endangered taxa, included in the Polish Red List of the algae in Poland (Siemińska et al. 2006). Four species from the endangered category were found (E): Eunotia botuliformis, Pinnularia nodosa, P. viridiformis and $P$. schoenfelderi.

Eunotia botuliformis has an optimum occurrence in silicate lowland streams, where it develops in large numbers. It prefers undisturbed anthropogenic, oligotrophic or dystrophic, less 
mineralized waters (Hofmann et al. 2011, LangeBertalot et al. 2011, 2017). Eunotia botuliformis is also known from Poland, from springs in the Eastern Tatra Mountain and the Beskid Sądecki (Wojtal 2013). It occurs individually in the Podkarpackie region in aquatic habitats (Noga et al. 2014b); so far, it has not been identified on soils.

Two Pinnularia species - P. schoenfelderi and $P$. viridiformis - are probably cosmopolitan diatoms, dispersed in oligo- to dystrophic waters, with low to medium electrolyte content (Krammer 2000, Lange-Bertalot et al. 2017). Pinnularia schoenfelderi is also less common in wet and humid places (Van Dam et al. 1994). They are known in Poland, where they occur individually in different types of waters, especially oligotrophic, and often in springs or upper sections of streams (Noga et al. 2014b, Pajączek et al. 2015). They are rarely found on soils - Pinnularia viridiformis always occurs singly (Stanek-Tarkowska et al. 2015, 2016), whereas P. schoenfelderi usually develops singly (Pajączek et al. 2015, StanekTarkowska et al. 2015, 2016); however, on sandyloam soils in Kosina near Rzeszów, it was numerous (5-20\%) and dominant (Stanek-Tarkowska et al. 2017). Both species were included on the Red List of the algae in Poland (Siemińska et al. 2006) as endangered (category E). However, the research conducted in recent years in south-eastern Poland shows that the species are commonly found in different types of habitats, usually individually, while $P$. schoenfelderi is more often developing outside the aquatic environment, especially on soils. For this reason, it appears that these diatoms should not be assigned to the category of endangered species, but recognized as rare species. However, further research is needed, especially in various terrestrial habitats, which would allow for changing the threat category and extending the ecological preferences of these species.

Pinnularia nodosa - a species widespread in Central Europe in flowing waters, peat bogs and ecosystems with low electrolyte content and $\mathrm{pH}$ below 5.5 - was also found in the endangered category (E). It rarely develops in large numbers, especially in periodically wet or humid environments (Van Dam et al. 1994, Krammer 2000, Hofmann et al. 2011, Lange-Bertalot et al. 2017). The species is also known from springs in central and southern Poland (Żelazna-Wieczorek 2011, Wojtal 2013) and from several sites in the Podkarpackie province (Noga et al. 2014a, b), but it was not reported in soils. This research shows that it can also develop in soil environments, especially on acidic and mossy soils.

An interesting species is Pinnularia cuneola, which occurred individually only on site R3. The species is known from a few locations in the world, including puddles in Germany, Alpine lakes and the USA (Krammer \& Lange-Bertalot 1986, Krammer 2000). Pinnularia cuneola is mentioned in the taxa lists from Romania (Caraus 2017) and the Netherlands (Veen et al. 2015). It was also found in a small puddle in Stalowa Wola (Rybak et al. 2019). Until now, the species has not been reported in soils, and studies have shown that it can also develop in this habitat, especially on sthe oils covered with mosses.

The conducted studies showed a large variety of diatom communities developing in the terrestrial environment, under the conditions of varied landscape use. The natural landscape of the Low Beskids is conducive to the occurrence of many common species, but is also a refuge for rare species, not yet recorded or even considered as endangered. The diatom studies and laboratory analyses of environmental parameters have shown a great need to expand the studies on the diatom communities of terrestrial habitats. Other authors also believe that the current studies conducted on the terrestrial diatom communities are still incomplete (Antonelli et al. 2017).

\section{Acknowledgments}

The author wishes to thank dr hab. Teresa Noga for any help with the manuscript.

The project was funded under the Ministry of Science and Higher Education under the name of "Regional Excellence Initiative" in the years 2019-2022 Project No. 026/RID/2018/19.

\section{REFERENCES}

1. Adesalu T.A., Olugbemi O.M. 2015. Soil algae: A case study of two vegetable farmlands in Lagos and Ogun states, southwest Nigeria. Ife Journal of Science, 17(3), 765-772.

2. Antonelli M., Wetzel C.E., Ector L., Teuling A.J., Pfister L. 2017. On the potential for terrestrial diatom communities and diatom indices to identify anthropic disturbance in soils. Ecological Indicators, 75, 73-81.

3. Banach A., Wolińska A., Błaszczyk M., Stępniewska Z. 2015. The influence of soil properties and land use on the phosphate level in soils from Lubelskie 
region Polish Journal of Agronomy, 22, 3-9.

4. Barragán C., Wetzel C.E., Ector L. 2018. A standard method for the routine sampling of terrestrial diatom communities for soil quality assessment. Journal of Applied Phycology, 30(2), 1095-1113.

5. Bérard A., Rimet F., Capowiez Y., Leboulanger C. 2004. Procedures for determining the pesticide sensitivity of indigenous soil algae: a possible bioindicator of soil contamination? Arch. Environ. Contam. Toxicol., 46, 24-31.

6. Cameron R.E. 1960. Communities of soil algae occuring in the Sonoran Desert in Arizona. Journal Arizona Acadademic Science, 1, 85-88.

7. Cameron R.E. 1962. Species of Nostoc Vaucher occuring in the Sonoran Desert in Arizona. Transactions of the American Microscopical Society, 81, 379-384.

8. Caraus I. 2017. Algae of Romania. A distributional checklist of actual algae. Version 2.4. Studii si Cercetari Biologie, 7, 1-1002.

9. Durrell L.W. 1959. Algae in Colorado soils. American Midland Naturalist, 61, 322-328.

10. Durrell L.W. 1962. Algae of Death Valley. Transactions of the American Microscopical Society, 81, 267-268.

11. Ettl H., Gärtner G. 1995. Sylabus der Boden-, Luftund Flechtenalgen. Gustaw Fischer, Stuttgart - Jena - New York.

12. Foets J., Wetzel C.E., Teuling A.J., Pfister L. 2020. Temporal and spatial variability of terrestrial diatoms at thecatchment scale: controls on communities. PeerJ, 8, e8296.

13. Gajda A.M., Przewłoka B. 2012. Soil biological activity as affected by tillage intensity. International Agrophisics, 26, 15-23.

14. Gollerbah M.M., Shtina E.A. 1969. Pochviennyje vodorosli. Nauka, Leningrad.

15. Hahn A., Neuhaus W. 1997. Boden-Diatomeen einer landwirtschaftlichen Nutzfläche bei Potsdam, Deutschland. Nova Hedwigia, 65(1-4), 285-298.

16. Heger T.J., Straub F., Mitchell E.A.D. 2012. Impact of farming practices on soil diatoms and testate amoebae: A pilot study in the DOK-trial at Therwil, Switzerland. Eur. J. Soil Biol., 49, 31-36.

17. Hofmann G., Werum M., Lange-Bertalot H. 2011. Diatomeen im Süsswasser-Benthos von Mitteleuropa. Bestimmungsflora Kieselalgen für die ökologische Praxis. Über 700 der häufigsten Arten und ihre Ökologie. [In:] H. Lange-Bertalot (Ed.), A.R.G. Ganter Verlag K.G., Königstein/Germany.

18. Hunt C.D., Durrell L.W. 1966. Distribution of fungi and algae. U.S. Geological Survey Professional Paper, 509, 55-66.

19. Ignaczak S. 1998. A maintenance system of fallow soil - changes in temperature, humidity and salinity of the various layers. Fragmenta Agronomica, 5, 225-237, (in Polish).

20. Jankowska-Huflejt H. 2007. Agri-environmental importance of permanent grasslands. Problems of Agricultural Engineering, 1, 23-34, (in Polish).

21. Johansen J.R., Shubert L.E. 2001. Algae in soils. Nova Hedwigia, Beiheft, 123, 297-306.

22. Kalinowska R., Pawlik-Skowrońska B. 2008. Metal resistance of soil algae (Chlorophyta) occurring in post-flotation $\mathrm{Zn} / \mathrm{Pb}$ - and $\mathrm{Cu}$-tailing ponds. Polish J. Ecol., 56(3), 415-430.

23. Karczewska A., Kabała C. 2008. Methodology of laboratory analysis of soils and plants, Methodology in force in the laboratories of the INGOŚ Environmental Protection Institute, Wrocław, (in Polish).

24. Kawecka B. 2012. Diatom diversity in streams of the Tatra National Park (Poland) as indicator of environmental conditions. Szafer Institute of Botany, Polish Academy of Sciences, Kraków.

25. Komárek J., Fott B. 1983. Chlorococcales. [In:] G. Huber Pestalozzi (Ed.), Das Phytoplankton des Süßwassers, 7(1), 283-308.

26. Krammer K. 2000. The genus Pinnularia [In:] H. Lange-Bertalot (Ed.) Diatoms of Europe. A.R.G. Gantner Verlag KG, Vaduz.

27. Krammer K., Lange-Bertalot H. 1986. Bacillariophyceae. 1. Naviculaceae. [In:] H. Ettl et al. (Eds), Süsswasserflora von Mitteleuropa. 2(1), G. Fischer Verlag, Stuttgart-New York.

28. Krammer K., Lange-Bertalot H. 1991. Bacillariophyceae. 3. Centrales, Fragilariaceae, Eunotiaceae. [In:] H. Ettl et al. (Eds), Süsswasserflora von Mitteleuropa. 2(3), G. Fischer Verlag, Stuttgart-Jena.

29. Krążel R. 1990. The dynamics of changes in the physical properties of sundy soil in different ways. Zesz. Probl. Post. Nauk Rol., 376, 25-30, (in Polish)

30. Lange-Bertalot H., Bąk M., Witkowski A. 2011. Eunotia and some related genera. [In:] Diatoms of Europe. Diatoms of the European inland water and comparable habitats. Vol. 6 H. Lange-Bertalot (Ed.), A.R.G. Gantner Verlag K.G., Ruggell.

31. Lange-Bertalot H., Hofmann, Werum M., Cantonati M. 2017. Freshwater benthic diatoms of Central Europe: over 800 common species used in ecological assessments. English edition with updated taxonomy and added species. [In:] M. Cantonati et al. (Eds), Koeltz Botanical Books, Schmitten-Oberreifenberg.

32. Noga T., Peszek Ł., Stanek-Tarkowska J., Pajączek A. 2014a. The Pinnularia genus in south-eastern Poland with consideration of rare and new taxa to Poland. Oceanological and Hydrobiological Studies, 43(1), 77-99.

33. Noga T., Kochman N., Peszek Ł., Stanek-Tarkowska J., Pajączek A. 2014b. Diatoms (Bacillariophyceae) 
in rivers and streams and on cultivated soils of the Podkarpacie Region in the years 2007-2011. Journal of Ecological Engineering, 15(1), 6-25.

34. Noga T., Stanek-Tarkowska J., Kochman-Kędziora N., Rybak M., Peszek Ł., Pajączek A. 2017. Luticola frequentissima Levkov, Metzeltin \& Pavlov - morphological and ecological characteristic of population from Southern Poland. Oceanological and Hydrobiological Studies, 46(2), 237-243.

35. Pajączek A., Peszek Ł., Kochman-Kędziora N., Rybak M., Noga T., Stanek-Tarkowska J. 2015. Occurrence of endangered diatom Pinnularia schoenfelderi Krammer on the area of south-eastern Poland. [In:] J. Gąsior et al. (Eds), Acta Carpathica, 23, 67-72 (in Polish with English summary).

36. Patoczka P. 2016. Skylines. University of Rzeszów, Rzeszów, (in Polish)

37. Pfister L., Wetzel C.E., Klaus J., Martínez-Carreras N., Antonelli M., Teuling A.J., McDonnell J.J. 2017. Terrestrial diatoms as tracers in catchment hydrology: a review. Wiley Interdisciplinary Reviews: Water, 4(6), p. 1241.

38. Pudełko J., Wright D., Wiatrak P. 1994. Applying restrictions on farming in the United States. Zeszyty Postępów Nauk Rolniczych, 1, 153-162 (in Polish).

39. Rybak M., Noga T., Zubel R. 2018. The aerophytic diatom assemblages developed on mosses covering the bark of Populus alba L. Journal of Ecological Engineering, 19(6), 113-123.

40. Rybak M., Noga T., Poradowska A. 2019. Diversity in anthropogenic environment - permanent puddle as a place for development of diatoms. Journal of Ecological Engineering, 20(8), 165-174.

41. Shields L.M., Drouet F. 1962. Distribution of terrestial algae within the Nevada Test Site. American Journal of Botany, 49, 457-554.

42. Siebielec G., Smreczak B., Klimkowicz-Pawlas A., Kowalik M., Kaczyński R., Koza P., Ukalska-Jaruga A., Łysiak M., Wójtowicz U., Poręba L., Chabros E. 2017. Report from the 3rd Stage of the Order Implementation "Monitoring of Soil Chemistry in Poland in 2015-2017'. Instytut Uprawy, Nawożenia i Gleboznawstwa PIB, Puławy (in Polish).

43. Sieminiak D. 1998. Biomass of soil algae under rye in different crop rotation systems. Polish Journal of Soil Science, 31(2), 79-85.

44. Sieminiak D. 2003. Soil algae in two farming systems of potato cropping. Bulletin of the Polish Academy of Sciences. Biological Sciences, 51(1), 1-8.

45. Siemińska J., Wołowski K. 2003. Catalogue of Polish prokaryotic and eucaryotic algae. W. Szafer Intitute of Botany, Polish Academy of Sciences, Kraków

46. Siemińska J., Bąk M., Dziedzic J., Gąbka M., Gregorowicz P., Mrozińska T., Pełechaty M., Owsiany P.
M., Pliński M. \& Witkowski A. 2006. Red list of the algae in Poland. [In:] Z. Mirek et al. (Eds), Red list of plants and fungi in Poland. W. Szafer Institute of Botany, Polish Academy of Sciences, Kraków

47. Skiba S. 2006. Soil cover of the alpine zone of the Carpathians and its threats. Roczniki Bieszczadzkie, 14, 201-214, (in Polish with English summary).

48. Stanek-Tarkowska J., Noga T. 2012a. The diatom communities developing on dust soils under sweet corn cultivation in Podkarpackie region. Fragmenta Floristica et Geobotanica Polonjca, 19(2), 525-536.

49. Stanek-Tarkowska J., Noga T. 2012b. Diversity of diatoms (Bacillariophyceae) in the soil under traditional tillage and reduced tillage. Inżynieria Ekologiczna, 30, 287-296.

50. Stanek-Tarkowska J., Noga T., Pajączek A., Peszek Ł. 2013. The occurrence of Sellaphora nana (Hust.) Lange-Bert. Cavacini, Tagliaventi \& Alfinito, Stauroneis borrichii (J.B. Petersen) J.W.G. Lund, S. parathermicola Lange-Bert. and S. thermicola (J.B. Petersen) J.W.G. Lund on agricultural soils. Algological Studies, 142, 109-120.

51. Stanek-Tarkowska J., Noga T., Kochman-Kędziora N., Peszek Ł., Pajączek A., Kozak E. 2015. The diversity of diatom assemblages developed on fallow soil in Pogórska Wola near Tarnów (southern Poland). Acta Agrobot., 68(1), 33-42.

52. Stanek-Tarkowska J., Noga T., Kochman-Kędziora N., Rybak M. 2016. Diatom assemblages growing on cropping soil in Pogórska Wola near Tarnów. Inżynieria Ekologiczna, 46, 128-134 (in Polish with English summary).

53. Stanek-Tarkowska J., Czyż E.A., Kaniuczak J., Poradowska A. 2017. Physicochemical properties of silt loamy soil and diversity of diatom species under winter wheat and oats. Journal of Ecological Engineering, 18(6), 142-151.

54. Stanek-Tarkowska J., Czyż E.A., Dexter A.R., Sławiński C. 2018. Effects of reduced and traditional tillage on soil properties and diversity of diatoms under winter wheat. International Agrophisics, 32, 403-409.

55. Szewczyk A. 2017. Impact of agricultural use on selected soil properties in the buffer zone of the Magura National Park. Ph. D. Thesis, University of Rzeszów, Rzeszów (in Polish with English summary).

56. Škaloud P. 2009. Species composition and diversity of aero-terrestial algae and Cyanobacteria of the Boreč Hill ventaroles. Fottea, 9(1), 65-80.

57. Ter Braak C.J.F., Šmilauer P. 2012. Canoco reference manual and user's guide. Software for ordination (version 5.0). Microcomputer Power, Ithaca, New York.

58. Van Dam H., Martens A., Sinkeldam J. 1994. A 
coded checklist and ecological indicator values of freshwater diatoms from the Netherlands. Netherlands Journal of Aquatic Ecology, 28(1), 117-133.

59. Veen A., Hof C.H.J., Kouwets F.A.C., Berkhout T. 2015. Taxa Watermanagement the Netherlands (TWN) [Rijkswaterstaat Waterdienst, Informatiehuis Water] http://ipt.nlbif.nl/ipt/ resource? $r=$ checklist-twn.

60. Wojtal A.Z. 2013. Species composition and distribution of diatom assemblages in spring waters from various geological formations in southern Poland. J. Cramer, Gebrüder Borntraeger Verlagsbuchhandlung, Stuttgart. Bibliotheca Diatomologica, 59, 1-436.

61. Włodek S., Pabin J., Biskupski A. 2007. The dynamics of soil water content at different methods of soil tillage. Fragmenta Agronomica, 24(4)4, 254-260 (in Polish with English summary).

62. Zancan S., Trevisan R., Paoletti M.G. 2006. Soil algae composition under different agro-ecosystems in North-Eastern Italy. Agriculture, Ecosystems and Environment, 112, 1-12.

63. Żelazna-Wieczorek J. 2011. Diatom flora in springs of Łódź Hills (Central Poland). Biodiversity, taxonomy and temporal changes of epipsammic diatom assemblages in springs affected by human impact. Diatom Monographs, 13, 1-420.

64. Żyszkowska M. 2003. Protected plant species and grassland habitats in the valley of a mountain river - the Bystrzyca Dusznicka River. Woda Środowisko Obszary Wiejskie, T. 6, 2(18), 387-396 (in Polish with English summary). 